\title{
Safety and Efficacy of Oral Flecainide Acetate in Patients with Gardiac Arrhythmias
}

\author{
Yuji NAKAZATO, MD, Yasuro NaKata, MD, \\ Masayuki YASUDA, MD, Kaoru NAKAZATO, MD, \\ Masataka Sumryoshi, MD, Shunsuke OGura, MD, \\ and Hiroshi Yamaguchi, MD
}

\section{SUMMARY}

We retrospectively studied the clinical efficacy and safety of oral flecainide in 38 patients with symptomatic arrhythmias. Patients received 100 to $200 \mathrm{mg}$ daily of flecainide for a mean of 25 months (range 8 to 50 months). All patients had normal cardiac function. Paroxysmal atrial fibrillation (PAF) was observed in 29 patients (76\%). Other forms of arrhythmia included paroxysmal atrial flutter, seen in 3 patients (8\%); premature atrial contraction and ventricular premature contraction, each seen in 2 patients $(5 \%)$; and supraventricular tachycardia and ventricular tachycardia, each seen in 1 patient $(3 \%)$.

A complete response was obtained in $15(52 \%)$ of 29 patients with PAF and a partial response in 8 patients (27\%). The remaining 6 patients $(21 \%)$ showed no response. A complete response was also obtained in 7 of 9 patients with other forms of arrhythmia. There were no differences in cardiac function and ECG parameters before and after treatment. Flecainide was withdrawn in 4 patients due to the development of electrocardiographic abnormalities. Three of these patients showed an atrial proarrhythmic effect. Abnormal ST elevations in the precordial leads were observed in 1 patient who received $200 \mathrm{mg}$ of flecainide daily.

In conclusion, flecainide was effective treatment for supraventricular and ventricular arrhythmias, but attention must be paid to the drug's potential proarrhythmic adverse effects. (Jpn Heart J 1997; 38: 379-385)

Key words: Flecainide, Proarrhythmia, ST elevation

YLASS Ic antiarrhythmic drugs are known to be effective treatment for
1 various arrhythmias. ${ }^{1-4)}$ However, CAST recently revealed that flecainide
and encainide had proarrhythmic and cardiosuppressive effects, ${ }^{5)}$ which could
limit the use of flecainide due to safety concerns. We investigated the clinical

From the Division of Cardiology, Department of Internal Medicine, Juntendo University School of Medicine, Tokyo, Japan.

Address for correspondence: Yuji Nakazato, MD, Division of Cardiology, Department of Internal Medicine, Juntendo University School of Medicine, 2-1-1, Hongo, Bunkyo-ku, Tokyo 113, Japan.

Received for publication October 9, 1996.

Accepted January 20, 1997. 
efficacy and safety of long-term treatment with flecainide in patients with various arrhythmias and normal cardiac function.

\section{Patients and Methods}

We retrospectively studied the safety and efficacy of flecainide in 38 patients with symptomatic supraventricular or ventricular arrhythmias who were treated with flecainide (Table I). In patients with paroxysmal tachyarrhythmias, the average incidence of attacks was 2 times per week with various durations. Patients received $100 \mathrm{mg}$ to $200 \mathrm{mg}$ daily of flecainide for a mean of 25 months (range 8 to 50 months). Patients with congestive heart failure, severely impaired left ventricular function, bradyarrhythmias, recent myocardial infarction, unstable angina, renal dysfunction, liver dysfunction and other serious illnesses were excluded.

Evaluation of safety: Their clinical findings were assessed and the patients underwent a physical examination at each visit. The interval between visits was one to two weeks after the initial administration of flecainide, and thereafter every one to two months. The PR, QRS, QT and QTc intervals were also measured on a 12-lead electrocardiogram (ECG) at each visit. Ambulatory Holter ECG was recorded every 3 to 6 months. Chest X-ray, echocardiography and routine blood test were performed during the follow-up period at appropriate intervals. The cardiothoracic ratio (CTR) was calculated from the chest X-ray and the ejection fraction (EF) was calculated by echocardiography. Cardiac and noncardiac side effects were assessed, including by laboratory test, at the baseline and treatment periods. The plasma concentration of flecainide was measured

Table I. Patient Characteristics

\begin{tabular}{lc}
\hline Number of patients & 38 \\
Male/Female & $32 / 6$ \\
Mean age (years) & 55 (range $32-76)$ \\
Mean follow-up (months) & 25 (range $8-50)$ \\
Daily dose (mg) & \\
100 & 15 \\
150 & 19 \\
200 & 4 \\
Underlying disease & \\
Hypertension & 4 \\
Sick sinus syndrome & 4 \\
WPW syndrome & 4 \\
Ischemic heart disease & 2 \\
Valvular heart disease & 1 \\
Alcoholic cardiornyopathy & 1 \\
Non-cardiac disease & 5 \\
None & 17 \\
\hline
\end{tabular}


during the follow-up period. Numeric values were compared between the baseline and the latest examination.

Evaluation of efficacy: The efficacy of flecainide was evaluated in terms of the improvement in subjective symptoms and changes in the parameters of standard and 24-hour ambulatory ECGs.

A complete response was defined as the absence of symptomatic arrhythmias and a partial response as a greater than $50 \%$ reduction in arrhythmias with tolerable symptoms.

Statistical analysis: Data are presented as the mean $\pm \mathrm{SD}$ and were evaluated by the paired t-test. A $p$ value $<0.05$ was regarded as significant.

\section{Results}

Arrhythmias and drug efficacy: Paroxysmal atrial fibrillation (PAF) was observed in 29 patients $(76 \%)$ and paroxysmal atrial flutter in 3 patients $(8 \%)$. Premature atrial contraction and premature ventricular contraction were each observed in 2 patients (5\%), and paroxysmal supraventricular tachycardia and ventricular tachycardia were each observed in 1 patient (3\%).

Of the 29 patients with PAF, 23 (79\%) responded to therapy. A complete response was achieved in 15 patients $(52 \%)$ and a partial response in 8 patients $(27 \%)$. The remaining 6 patients $(21 \%)$ showed no response. Flecainide was administered to 3 patients in the complete response group during attacks of atrial fibrillation and resulted in conversion to sinus rhythm. Flecainide resulted in a complete response in 2 of 3 patients with paroxysmal atrial flutter; 1 of these patients showed conversion to sinus rhythm. In this case of converted sinus rhythm, atrial fibrillation or flutter was confirmed to last at least one week; therefore, it was considered to have been due to the effect of flecainide rather than a spontaneous termination. The 5 patients with premature atrial contraction, premature ventricular contraction, or paroxysmal supraventricular tachycardia achieved complete responses. Ventricular tachycardia disappeared after treatment in the patient with this type of arrhythmia, although coupled premature ventricular contractions persisted.

Cardiac function and ECG parameters: There were no significant differences in parameters of cardiac function and ECG before and after treatment (Table II).

The mean plasma concentration of flecainide during the follow-up period was $239.6 \pm 112.5 \mu \mathrm{g} / \mathrm{m} l$. It was $280 \mathrm{ng} / \mathrm{m} l$ in the responders and $201 \mathrm{ng} / \mathrm{m} l$ in non-responders.

Side effect: Flecainide was withdrawn in 4 patients due to the development of electrocardiographic abnormalities. One patient developed ectopic atrial 
Table II. Cardiac Function and ECG Parameters

\begin{tabular}{llll}
\hline & & Baseline & Follow-up \\
\hline CTR $(\%)$ & $48.5 \pm 3.9$ & $49.7 \pm 7.4$ \\
EF $(\%)$ & $69.4 \pm 5.8$ & $70 \pm 7.5$ \\
ECG & & \\
R-R & (sec) & $0.95 \pm 0.17$ & $0.97 \pm 0.18$ \\
P-R & $(\mathrm{sec})$ & $0.18 \pm 0.03$ & $0.19 \pm 0.03$ \\
QRS & (sec) & $0.08 \pm 0.01$ & $0.09 \pm 0.01$ \\
QT & $(\mathrm{sec})$ & $0.4 \pm 0.08$ & $0.4 \pm 0.03$ \\
QTc & $0.42 \pm 0.04$ & $0.42 \pm 0.03$ \\
\hline
\end{tabular}

$\mathrm{CTR}=$ cardiothoracic ratio; $\mathrm{EF}=$ ejection fracton .
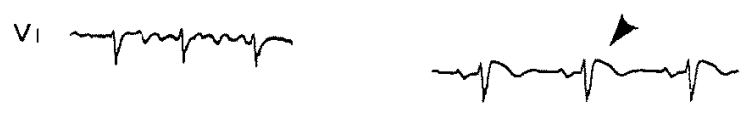<smiles>CC(C)CC(C)CC(C)CC(C)C</smiles><smiles>[CH]C</smiles><smiles>CCCCC(C)(C)CC(C)CCC=C(C)C</smiles>

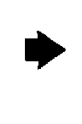<smiles>CC(CC(C)(C)C)CC(C)(C)C(C)(C)C</smiles><smiles>CC(C)(C)CC(C)(C)CC(C)(C)CC(C)(C)C</smiles>

\section{Pre-treatment}

\section{Flecainide $200 \mathrm{mg} / \mathrm{day}$}

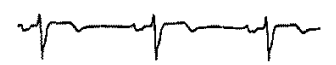<smiles>CCCCCCC(C)CCC</smiles><smiles>CCCCCCC(C)CCC(C)CCCC</smiles>

Post-treatment

Figure. EGG change attributed to the adverse effect of flecainide in a patient with paroxysmal atrial fibrillation. Although sinus rhythm was restored after 2 weeks of treatment with flecainide, ST elevations in leads $V_{1}$ to $V_{3}$ were observed. Discontinuation of flecainide resulted in normalization of the ST segment.

tachycardia associated with atrioventricular block and 2 patients with PAF showed persistent atrial flutter with the reduction of heart rate. One patient showed a rate of $130 / \mathrm{min}$ with 2:1 ventricular conduction. Another patient showed the heart rate of $64 / \mathrm{min}$ with 2:1 to $4: 1$ ventricular conduction. Abnormal ST elevations in precordial leads were observed in 1 patient who received $200 \mathrm{mg}$ daily of flecainide. This electrocardiographic abnormality was eliminated by discontinuation of the drug (Figure). One patient complained of lightheadedness after treatment, but this effect was well tolerated and the plasma concentration was $281 \mathrm{ng} / \mathrm{ml}$. No other non-cardiac adverse effects were observed. 


\section{Discussion}

Flecainide acetate is a class Ic antiarrhythmic drug that has been found to be effective in treating patients with various arrhythmias. ${ }^{1-4)}$ However, the results of CAST suggested that flecainide has proarrhythmic and cardiosuppressive effects. ${ }^{5)}$ The present results indicate that long-term treatment with flecainide is safe and effective in patients with normal cardiac function.

Clinical efficacy of flecainide: Flecainide, is especially effective in patients with supraventricular arrhythmias, including PAF. ${ }^{1-3)}$ We consider the effects of flecainide on supraventricular arrhythmia should be emphasized more strongly. PAF and paroxysmal atrial flutter were the most common arrhythmias in the present retrospcctive study. Completc and partial responses were achieved in 23 (79\%) of 29 patients with PAF and in 2 of 3 paticnts with atrial fluttcr. Pictersen et al. ${ }^{6}$ reported that flecainide completely suppressed PAF and paroxysmal atrial flutter in $15(35 \%)$ of 43 patients after 1 week treatment, in $18(46 \%)$ of 39 patients after 1 month treatment, and in $12(50 \%)$ of 24 patients after 3 month treatment. Although adverse effects were reported in 32 (74\%) of 43 patients, most effects were tolerable and treatment was withdrawn in only 2 patients. Sihm et al. ${ }^{7}$ reported that flecainide-induced conversion to sinus rhythm was achieved in $21(49 \%)$ of 43 patients with atrial fibrillation and $10(34 \%)$ of 29 patients with atrial flutter.

The number of patients with other arrhythmias in the present study was too small to permit a comparison with the results of previous studies. However, these arrhythmias were suppressed in 5 of 6 patients.

Adverse effects of flecainide: The major concern related to the use of class Ic drugs is their adverse effects. Previous studies have shown that although noncardiac adverse effects are common, they are usually tolerable. ${ }^{6,8)}$ However, Sihm et al. ${ }^{7}$ observed severe arrhythmogenic events in 9 of 100 patients; this effect was most common in patients with ischemic heart disease and in elderly patients. Brembilla-Perrot et al. ${ }^{\text {9) }}$ found that an ejection fraction of less than $30 \%$ was a predisposing factor for adverse effects.

In the present study, 4 patients showed ECG changes during flecainide treatment. The development of persistent atrial flutter was observed in 2 patients with PAF. Although the heart rate was reduced due to the prolongation of the atrial interval and the decrease in ventricular conduction, flecainide enhanced the conduction disturbance in the atrium and appeared to maintain reentry in these patients. One patient showed paroxysmal atrial tachycardia associated with atrioventricular block. These effects appeared to represent the atrial proarrhythmic adverse effects of flecainide. Similar findings were observed in previous studies of propafenon and flecainide. ${ }^{(0,11)}$ 
An unusual EGG finding was observed in a 58-year-old male patient with PAF. Abnormal ST elevations were detected in precordial leads $V_{1}$ to $V_{3}$ after treatment with $200 \mathrm{mg}$ daily of flecainide. This change was similar to the ST elevation observed by Brugada and Brugada in patients with idiopathic ventricular fibrillation. ${ }^{12)}$ The ST-segment elevation disappeared after flecainide was discontinued in our patients. We discontinued flecainide even though no fatal ventricular arrhythmia occurred. Other factors relevant to the ST elevation, such as coronary vasospasm, were excluded. We concluded that the ST-segment elevation was due to the adverse effect of flecainide. ${ }^{13}$ )

Crijns et al. ${ }^{14)}$ observed marked prolongation of the QRS complex, mimicking ventricular tachycardia, in a patient receiving flecainide. Aubel et al. ${ }^{15)}$ observed a pseudo-myocardial infarction ECG pattern induced by flecainide. However, there are no previous reports of a ST elevation similar to that observed in the present study. Although the autonomic nervous system or a disorder in the His-Purkinje system may be involved, the mechanism of ST elevation is still unclear. ${ }^{12)}$ Miyazaki et al. ${ }^{16)}$ reported that the ST segment was elevated by class Ia drugs in patients with Brugada's syndrome. They suggested that the presence of an area of early repolarization or of local depolarization in the ventricle may cause this ST-segment elevation in patients with this syndrome. It was unclear whether a similar mechanism could be applied to our patient because the baseline condition was already different from Brugada's syndrome. Further study is needed to clarify the relation between ST-segment elevation and arrhythmia. Although the detailed ECG findings of patients with sudden death were not clarified in CAST, the higher mortality rate in the flecainide group compared with the placebo group suggests that there might be some relation between ST elevation and a proarrhythmic response.

Atrial proarrhythmic adverse effects were seen in 4 patients in the present study, but only 1 patient reported lightheadedness, which was tolerable. The low rate of adverse effects in the present study may have been related to the subjects' normal ejection fraction (average: $70 \%$ ), the small number of cases of ischemic heart disease ( 2 patients) and lower age (mean age: 55 years). In addition, the relatively lower plasma concentration of flecainide might have been a factor in the incidence of adverse effects, which was at the lower end of the normal range even among the patients who responded.

In conclusion, flecainide was effective in patients with supraventricular and ventricular arrhythmias with normal ventricular function, but attention must be paid to the drug's potential proarrhythmic adverse effects. 


\section{REFERENCES}

1. Prichett ELC, DaTorre SD, Platt ML, McCarville SE, Hougham AJ and The Flecainide Supraventricular Tachycardia Study Group. Flecainide acetate treatment of paroxysmal supraventricular tachycardia and paroxysmal atrial fibrillation: dose-response studies. J Am Coll Cardiol 1991; 17: 297-303.

2. Wiseman MN, Elstob JE, Camm AJ, Nathan AW. A study of the use of flecainide acetate in the longterm management of cardiac arrhythmias. Pacing Clin Electrophysiol 1990; 13: 767-75.

3. Neuss $\mathrm{H}$, Schlepper $\mathrm{M}$. Long-term efficacy and safety of flecainide for supraventricular tachycardia. Am J Cardiol 1988; 62: 56D-61D.

4. Facchini M, Varisco T, Bonazzi O, Priori SG, Songa V, Schwartz PJ. Efficacy and safety of flecainide in low-risk patients with chronic ventricular arrhythmias: a two-year follow-up. Am Heart J 1989; 117 : $1258-64$

5. GAST Investigators. Preliminary report: effect of encainide and flecainide on mortality in a randomized trial of arrhythmia suppression after myocardial infarction. N Engl J Med 1989; 321: 406-12.

6. Pietersen AH, Hellemann H, The Danish-Norwegian Flecainide Multicenter Study Group. Usefulness of flecainide for prevention of paroxysmal atrial fibrillation and futter. Am J Cardiol 1991; 67: 713-7.

7. Sihm I, Rasmussen HJ, Pedersen AK, Thygesen K. Flecainide acetate in atrial flutter and fibrillation. Eur Heart J 1990; 11: 145-8.

8. Hohnloser SH, Zabel M. Short- and long-term efficacy and safety of flecainide acetate for supraventricular arrhythmias. Am J Cardiol 1992; 70: 3A-10A.

9. Brembilla-Perrot B, Amor M, Auque F, et al. Effect of flecainide on left ventricular ejection fraction. Eur Heart J 1987; 8: 754-81.

10. Feld GK, Chen PS, Nicod P, Fleck RP, Meyer D. Possible atrial proarrhythmic effects of class lc antiarrhythmic drugs. Am J Cardiol 1990; 66: 378-83.

11. Berns E, Rinkenberger RL, Keang MK, Dougherty AH, Jenkins M, Naccarelli GV. Efficacy and safety of flecainide acetate for atrial tachycardia or fibrillation. Am J Cardiol 1987; 59: 1337-41.

12. Brugada P, Brugada J. Right bundle branch block, persistent ST segment elevation and sudden cardiac death: a distinct clinical and electrocardiographic syndrome. A multicenter report. J Am Coll Cardiol 1992; 62: 1303-6.

13. Nakazato $\mathrm{Y}$, Nakata $\mathrm{Y}$, Aihara $\mathrm{K}$, et al. The anti- and pro-arrhythmic effects of flecainide during the treatment for paroxysmal atrial flutter and fibrillation. Ther Res 1996; 17: 311-5. (in Japanese)

14. Crijns HJ, Gelder IC, Lie KI. Supraventricular tachycardia mimicking ventricular tachycardia during flecainide treatment. Am J Cardiol 1986; 62: 1303-6.

15. Aubel KJJCM, Ruiter JH, Arnold AER, Burgersdijk C. Pseudo infarction ECG pattern occurring during intravenous treatment with flecainide acetate. Eur Heart J 1992; 13: 137-9.

16. Miyazaki T, Mitamura H, Miyoshi S, Soejima K, Aizawa Y, Ogawa S. Autonomic and antiarrhythmic drug modulation of ST segment elevation in patients with Brugada syndrome. J Am Coll Cardiol 1996; 27; 1061-70. 